\title{
CHARACTERIZATION OF THE DAILY OXYTOCIN RHYTHM IN PRIMATE CEREBROSPINAL FLUID ${ }^{1}$
}

\author{
H. G. ARTMAN, ${ }^{2}$ S. M. REPPERT, ${ }^{*}$ M. J. PERLOW, $\ddagger$ S. SWAMINATHAN, T. H. ODDIE, AND \\ D. A. FISHER
}

Department of Pediatrics, UCLA School of Medicine, The Division of Endocrinology, Harbor-UCLA Medical Center, Torrance, California 90509, *Children's Service and Department of Neurology, Massachusetts General Hospital, Harvard Medical School, Boston, Massachusetts 02114, and $\ddagger$ Department of Neurology, Mount Sinai School of Medicine, New York, New York 10029

Received September 14, 1981; Revised December 21, 1981; Accepted December 22, 1981

\begin{abstract}
The circadian characteristics of the daily rhythm in oxytocin (OT) concentrations in cerebrospinal fluid (CSF) were studied in the rhesus monkey. Monkeys subjected to constant light or constant dark for periods of 3 to 6 days manifested persistence of the CSF OT rhythm. A 12-hr phase shift in the light-dark cycle resulted in a resynchronization of the rhythm to the new lighting schedule within 3 to 4 days. Altering the daily feeding and care schedule during a period of constant darkness did not alter the expression or timing of the CSF OT rhythm significantly. These results suggest that the OT rhythm is endogenously generated and that the daily light-dark cycle normally synchronizes the rhythm to the 24 -hr cycle.
\end{abstract}

Recently, we reported that there is a large daily rhythm in the oxytocin (OT) concentration in cerebrospinal fluid (CSF) of male rhesus monkeys (Perlow et al., 1981). Daytime CSF OT levels are 3- to 12-fold higher than night-time values. Interestingly, the CSF rhythm is not accompanied by a diurnal pattern of plasma OT concentrations. The presence of an OT rhythm confined to CSF suggests that the CSF is a vehicle for OT distribution to different parts of the brain where the peptide may have important neuromodulator functions.

In the present study, we sought to determine whether the CSF OT rhythm is generated endogenously and whether the daily light-dark cycle functions to coordinate the CSF rhythm to the solar day.

\section{Materials and Methods}

The monkey model for these experiments has been described previously in detail (Reppert et al., 1979). Briefly, adult male rhesus monkeys weighing 5.5 to 6.5

\footnotetext{
${ }^{1}$ The expert technical assistance of Ms. Rubye Lawrence is appreciated. We also thank Ms. Sharon Schuler and Ms. Mary Towles for their excellent secretarial work. This work was supported by Public Health Service Grants HD-06335 and HD-14427 from the National Institute of Child Health and Human Development. S. M. R. is a Research Fellow of the Charles A. King Trust, Boston, MA.

${ }^{2}$ To whom correspondence should be addressed at Research and Education Institute, Building A-17, 1124 West Carson Street, Torrance, CA 90502 .
}

$\mathrm{kg}$ were adapted to primate chairs. Each animal was kept in a separate, sound-attenuated isolation chamber; the chambers were cleaned between 10 A.M. and 12 noon. Food was placed on the feeding trays between 10 A.M. and 12 noon unless otherwise specified and remained available until the following day; this was done for experimental convenience and not as a means to restrict. feeding to a particular time of day. Water was available ad libitum. The diurnal lighting schedule consisted of 12 hr of light per day (LD 12:12), with lights on from 6 A.M. to 6 P.M.

CSF was withdrawn continuously at a rate of approximately $1 \mathrm{ml} / \mathrm{hr}$ from an indwelling polyethylene catheter terminating in the subarachnoid space at the high cervical to low cisternal region. CSF was collected in 2-hr fractions by an automated fraction collector. Samples remained at room temperature for approximately $2 \mathrm{hr}$ prior to refrigeration at $4^{\circ} \mathrm{C}$. Fractions were collected in the morning and frozen at $-20^{\circ} \mathrm{C}$ until assayed.

The double antibody OT radioimmunoassay (RIA) system has been described previously; $100 \mu \mathrm{l}$ of unextracted CSF were assayed in each tube (Weitzman et al., 1978). United States Pharmacopeia posterior pituitary reference material (2.4 U.S.P. posterior pituitary units of oxytocic activity/mg) was used as the standard in the RIA. All samples from a single experiment in an individual monkey were run in the same assay. Sensitivity of the RIA was defined as the amount of OT which would displace $10 \%$ of the ${ }^{125}$ I-OT in the assay tube. Samples 
which displaced less than $10 \%$ of label $\left(\mathrm{B}_{90}\right)$ were assigned the $B_{90}$ value for illustrative purposes; for statistical analysis, these values were deleted. Recoveries of 15 or 25 microunits $/ \mathrm{ml}$ of OT added to a monkey CSF pool ranged from 74 to $89 \%$. Dilution of monkey CSF samples showed parallelism with standard material in the buffer. Twenty-four-hour exposure of CSF to room temperature did not result in significant degradation of OT immunoreactivity.

The observed data were fitted to periodic curves; the OT level $y$ was computed as a sine function of the time $x$ hours from the beginning of each experiment. An iterative computer procedure was used to estimate the values of parameters to fit the data for each animal; the values of parameters were defined such that the sum of squares of differences between the computed and observed values of $y$ approached a minimum. The formulae used and the computed results are given in the appendix.

\section{Results}

Effects of constant light and dark on OT concentration. Four monkeys were sampled continuously in diurnal lighting (LD 12:12) for 3 to 4 days, followed by 3 to 4 days of constant light and a further 3 to 4 days of constant dark (Fig. 1). In diurnal lighting, all animals exhibited a marked increase in CSF OT concentration during daylight hours relative to the period of dark; OT concentrations began to increase before the onset of the light period and began to fall prior to the onset of the dark period. The peak OT levels varied from day to day in the same animal and between animals both in diurnal lighting and constant lighting conditions. However, the general pattern of OT rhythmicity persisted in all animals during the periods of constant light and constant dark. There were occasional deviations from this usual pattern of OT in CSF; these occurred both in diurnal lighting and under constant lighting conditions. For example, on the 3 rd day of diurnal lighting for animal 641 , the usual fall in OT was observed prior to the dark period, but this was followed by an additional 4- to $6-\mathrm{hr}$ period of OT concentrations comparable to those usually found during the daylight hours.

Effects of a 12-hr phase shift in food delivery and daily care. Animals were sampled after a 12-hr phase shift in food delivery and daily care to determine whether the daily $\mathrm{OT}$ rhythm in constant lighting conditions (i.e., constant dark) was artificially generated or influenced by the daily care and feeding system used in our paradigm. For this aspect of the study, two monkeys, 477 and 585, were sampled in diurnal lighting with daily care and food delivery at $10 \mathrm{~A}$.M. to 12 noon. The animals then were placed in constant dark with food delivery and daily care shifted by $12 \mathrm{hr}$ (Fig. 2). The OT rhythm observed during diurnal lighting was similar to that seen in Figure 1. Moreover, visual inspection of the data showed that the rhythm persisted during constant dark and during a 12-

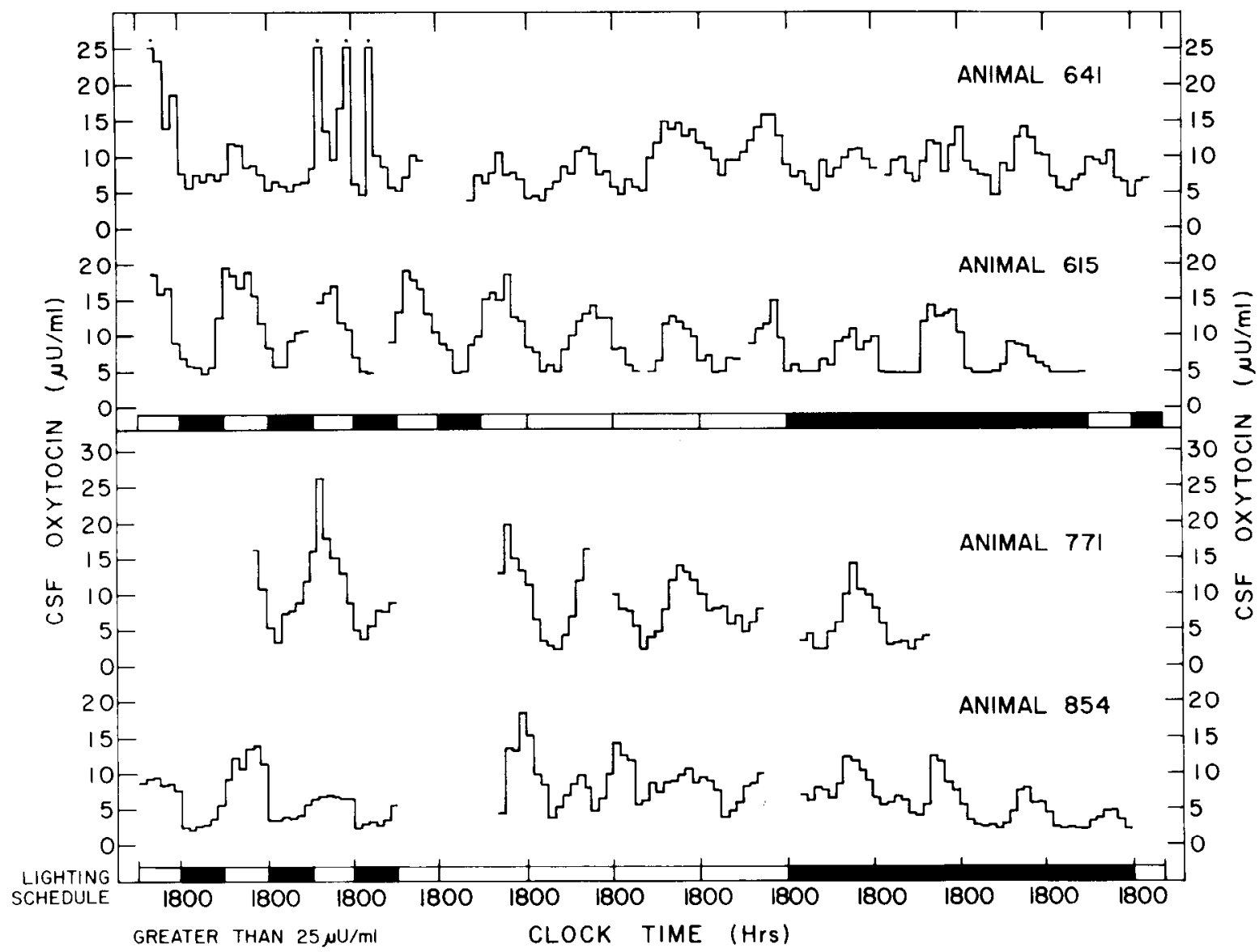

Figure 1. Pattern of CSF OT for four animals in diurnal lighting (LD 12:12) followed by continuous light (open bars) and continuous dark (solid bars). CSF samples were collected in 2 -hr aliquots. 
hr shift in feeding and daily care without any obvious was confirmed by the computer analysis outlined in alterations in the timing of the rhythm.

The apparent lack of effect of altering the daily care Tables I and II and Figure 3. The estimated parameters and equations tabulated in Table I yield computed peak and food cycle on the timing of the OT rhythm in dark

OT levels at the times shown in Table II. These times

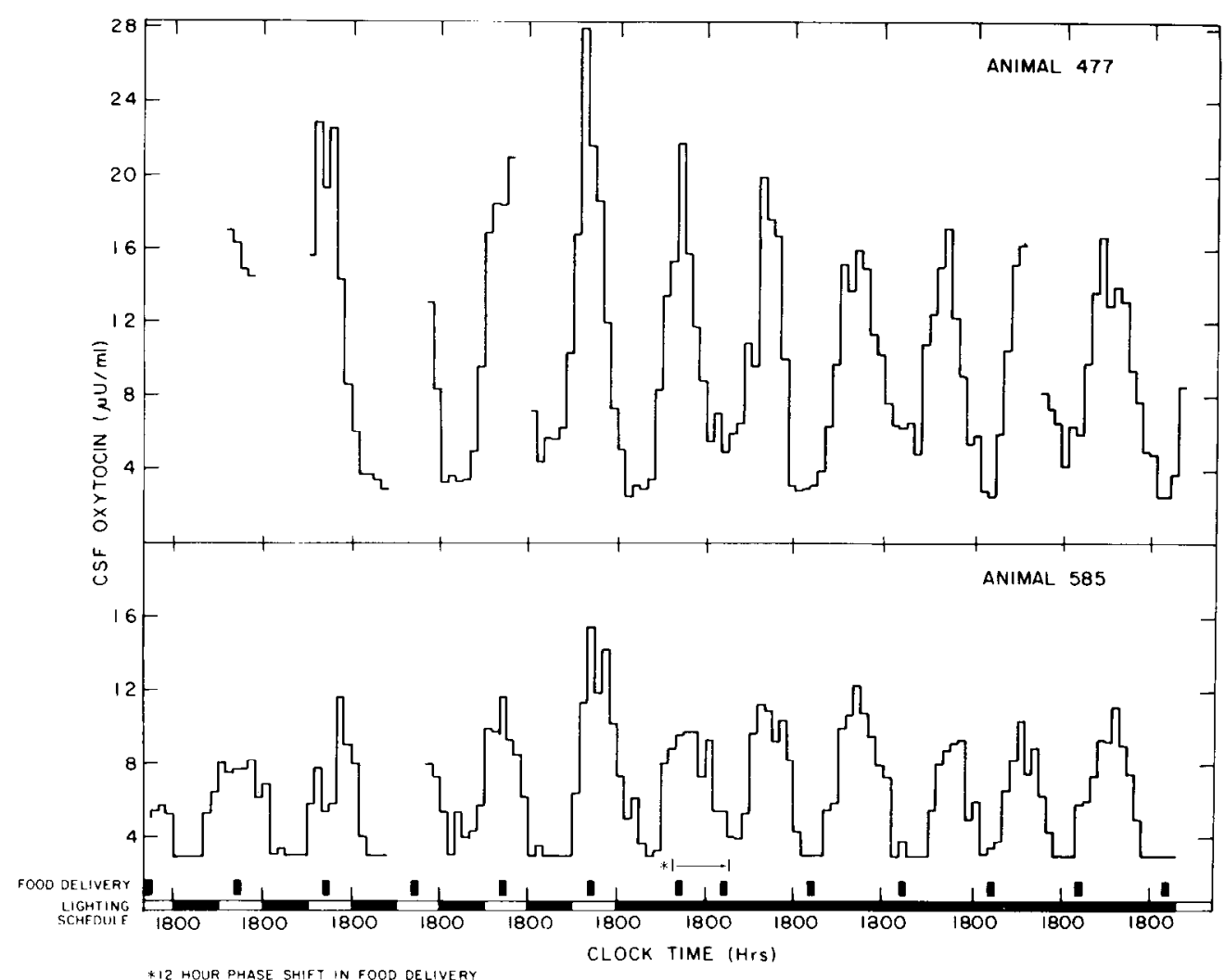

Figure 2. The pattern of CSF OT concentrations in two animals studied during a 12-hr phase reversal of the daily delivery of food and care in constant dark (solid bars). After several days in diurnal lighting, during which time daily care and food were delivered between 10 A.M. and 12 noon, the animals were placed in constant dark; the daily delivery of food and care was altered and maintained between 10 P.M. and 12 midnight. CSF was collected in 2-hr aliquols.

TABLE I

Results of computer fitting of observed OT level y to periodic sine functions of time $x$

\begin{tabular}{|c|c|c|c|c|c|c|c|c|c|}
\hline \multirow{2}{*}{$\begin{array}{l}\text { Animal and } \\
\text { Data Set }\end{array}$} & \multirow{2}{*}{$\begin{array}{l}\text { Equa- } \\
\text { tion }\end{array}$} & \multirow{2}{*}{$\begin{array}{l}\text { No. of } \\
\text { Obser- } \\
\text { vations }\end{array}$} & \multicolumn{6}{|c|}{ Estimated Values of Coefficients and SE } & \multirow{2}{*}{$\begin{array}{l}\text { SE of } \\
\text { the } \\
\text { Esti- } \\
\text { mate }\end{array}$} \\
\hline & & & $A$ & $B$ & $O$ or $O_{2}$ & $T$ & $O_{1}$ & E & \\
\hline $\begin{array}{l}\text { 477: } 0-140 \mathrm{hr} \text { normal food } \\
\text { schedule; diurnal lighting }\end{array}$ & 1 & 45 & $10.80 \pm 0.39$ & $9.04 \pm 0.54$ & $0.87 \pm 0.06$ & $24.00 \pm 0.03$ & & & 2.5 \\
\hline $\begin{array}{l}\text { 477: over } 140 \mathrm{hr} \text { reversed } \\
\text { food schedule; constant } \\
\text { dark }\end{array}$ & 1 & 71 & $9.20 \pm 0.37$ & $5.41 \pm 0.55$ & $-0.17 \pm 0.10$ & $23.40 \pm 0.02$ & & & 3.0 \\
\hline $\begin{array}{l}\text { 585: } 0-140 \mathrm{hr} \text { normal food } \\
\text { schedule; diurnal lighting }\end{array}$ & 3 & 48 & $4.88 \pm 0.29$ & $3.05 \pm 0.42$ & $0.96 \pm 0.13$ & $24.50 \pm 0.03$ & & $0.024 \pm 0.003$ & 1.9 \\
\hline $\begin{array}{l}\text { 585: over } 140 \mathrm{hr} \text { reversed } \\
\text { food schedule; constant } \\
\text { dark }\end{array}$ & 3 & 55 & $8.71 \pm 0.20$ & $2.95 \pm 0.28$ & $-1.18 \pm 0.09$ & $23.10 \pm 0.02$ & & $-0.008 \pm 0.001$ & 1.4 \\
\hline $\begin{array}{l}\text { 477: } 0-170 \mathrm{hr} \text { diurnal light- } \\
\text { ing }\end{array}$ & 1 & 62 & $12.74 \pm 0.43$ & $7.97 \pm 0.63$ & $-0.42 \pm 0.07$ & $24.30 \pm 0.04$ & & & 3.3 \\
\hline $\begin{array}{l}\text { 477: over } 170 \mathrm{hr} \text { reversed } \\
\text { light and food }\end{array}$ & $5^{a}$ & 71 & $13.83 \pm 0.29$ & $6.55 \pm 0.42$ & $-2.38 \pm 0.07$ & $24.44 \pm 0.01$ & $2.51 \pm 0.20$ & $0.045 \pm 0.003$ & 2.4 \\
\hline $\begin{array}{l}\text { 585: } 0-170 \mathrm{hr} \text { diurnal light- } \\
\text { ing }\end{array}$ & 1 & 83 & $11.80 \pm 0.33$ & $6.10 \pm 0.47$ & $-0.95 \pm 0.07$ & $23.95 \pm 0.04$ & & & 2.9 \\
\hline $\begin{array}{l}\text { 585: over } 170 \mathrm{hr} \text { reversed } \\
\text { light and food }\end{array}$ & $5^{a}$ & 64 & $7.39 \pm 0.23$ & $2.00 \pm 0.31$ & $-4.50 \pm 0.08$ & $23.96 \pm 0.03$ & $0.33 \pm 0.59$ & $-0.049 \pm 0.008$ & 1.7 \\
\hline
\end{tabular}

${ }^{a}$ Value $x_{0}=170 \mathrm{hr}$ used in equation 5. 
TABLE II

Calculated peak times of observed OT levels related to mid-times of the light period

\begin{tabular}{|c|c|c|c|c|}
\hline \multirow{2}{*}{ Treatment } & \multicolumn{2}{|c|}{ Monkey 477} & \multicolumn{2}{|c|}{ Monkey 585} \\
\hline & Mid-light & Peak OT & Mid-light & Peak OT \\
\hline & \multicolumn{2}{|c|}{$h r$} & \multicolumn{2}{|c|}{$h r$} \\
\hline \multirow{6}{*}{$\begin{array}{l}\text { Diurnal lighting; normal } \\
\text { food schedule; } 0-140 \mathrm{hr}\end{array}$} & 2 & 0.7 & 2 & 0.3 \\
\hline & 26 & 24.7 & 26 & 24.3 \\
\hline & 50 & 48.7 & 50 & 49.3 \\
\hline & 74 & 72.7 & 74 & 73.8 \\
\hline & 98 & 96.7 & 98 & 98.3 \\
\hline & 122 & 120.7 & 122 & 122.8 \\
\hline \multirow{7}{*}{$\begin{array}{l}\text { Reversed food schedule; } \\
\text { no light; over } 140 \mathrm{hr}\end{array}$} & 146 & 144.9 & 146 & 146.8 \\
\hline & 170 & 168.3 & 170 & 169.9 \\
\hline & 194 & 191.7 & 194 & 193.0 \\
\hline & $218^{a}$ & 215.1 & $218^{\alpha}$ & 216.1 \\
\hline & 242 & 238.5 & 242 & 239.1 \\
\hline & 266 & 261.9 & 266 & 263.3 \\
\hline & 290 & 286.2 & & \\
\hline \multirow{8}{*}{$\begin{array}{l}\text { Diurnal lighting schedule; } \\
0-170 \mathrm{hr}\end{array}$} & 8 & 5.7 & 8 & 7.6 \\
\hline & 32 & 30.0 & 32 & 31.5 \\
\hline & 56 & 54.3 & 56 & 55.5 \\
\hline & 80 & 78.6 & 80 & 79.4 \\
\hline & 104 & 102.9 & 104 & 103.3 \\
\hline & 128 & 127.2 & 128 & 127.1 \\
\hline & 152 & 151.5 & 152 & 151.1 \\
\hline & $(188)^{b}$ & $(175.8)^{c}$ & $(188)^{b}$ & $(175.1)^{c}$ \\
\hline \multirow{6}{*}{$\begin{array}{l}\text { Reversed light and food } \\
\text { schedule; over } 170 \mathrm{hr}\end{array}$} & 212 & 205.2 & 188 & 173.0 \\
\hline & 236 & 232.2 & 212 & 209.4 \\
\hline & 260 & 257.4 & 236 & 235.1 \\
\hline & 284 & 282.1 & 260 & 259.2 \\
\hline & 308 & 306.6 & 284 & 283.5 \\
\hline & & & 308 & 307.4 \\
\hline
\end{tabular}

\footnotetext{
${ }^{a}$ 24-hr intervals after light ceased

"Transitional period; see Figure 3.

" Extrapolated values.
}

agree closely with the mid-light time or the mid-light time expected by adding 24-hr intervals to the time when the no light period began. That is, the reversed food schedule had little effect on the timing of the peak CSF OT concentrations.

Effects of a 12-hr phase shift in lighting schedule, food delivery, and daily care. Two monkeys were sampled during 6 days of diurnal lighting followed by a 12-hr phase shift in lighting, food delivery, and daily care (monkeys 477 and 585). In these animals, the expected diurnal CSF OT concentration pattern was observed prior to the 12-hr phase shift (Fig. 4). Initially, after the 12-hr extension of the dark period, the CSF OT rhythm persisted as in constant dark. During the following 2 to 3 days, there was a transition period where the OT secretion was blunted and the duration of the peak levels was lengthened. Thereafter, the OT rhythm displayed the same phase relationship to light and dark in the reversed lighting schedule as normally occurs in diurnal lighting. The resynchronized pattern continued for the subsequent 3 days that the animals were sampled.

The pattern of resynchronization of the OT rhythm was examined by computer analysis (see "Appendix," Tables I and II, and Fig. 4). The computed parameters are shown in Table I. Table II again compares the computed peak times with the mid-light times and demonstrates how the phase of the sine curve (equation 5, "Appendix") changes from $O$, during the diurnal light period to $\mathrm{O}_{2}$ in the reversed light period, confirming the visual observation that it takes a few cycles for the rhythm to catch up with the sudden change in lighting schedule.

\section{Discussion}

The results of our study demonstrate the presence of a daily rhythm in the CSF OT concentration of the rhesus monkey. Furthermore, the data provide two lines of evidence which suggest that this daily rhythm in the CSF is an endogenously generated (i.e., circadian) rhythm. First, the daily rhythm persists, albeit for relatively short periods, in either constant light or constant dark. It is important to note that the rhythm does not appear to be influenced in a constant lighting condition (constant dark; Fig. 2) by the daily care and food delivery system used in these studies. Thus, the persistence of the rhythm in our constant dark environment means that the expression of the rhythm is not dependent on any discernible cycle alteration in the environment.

The second line of evidence in favor of the circadian nature of the OT rhythm is that the rhythm does not resynchronize immediately to a phase change in the lighting cycle. Instead, a few days are required for complete resynchronization to occur, a situation similar to that described for other circadian rhythms (Zucker, 1980). This finding also indicates that the daily light-dark cycle is the environmental stimulus which normally coordinates the CSF OT rhythm to the $24-\mathrm{hr}$ period.

The source of OT in CSF is not clear. The highly organized rhythmic pattern of OT concentrations confined to CSF suggests that CSF OT is derived via active secretion rather than from axonal diffusion or from transport from the blood to CSF. There is accumulating anatomical evidence of neuronal fiber sources of OT other than the classical magnocellular neuronal terminals in the posterior pituitary. Free nerve endings containing large secretory granules typical of nerve endings of the magnocellular neurons have been found in the floor of the third ventricle (Scott et al., 1974). In addition, multiple projections of OT-containing fibers from the paraventricular nuclei have been described, and OT-containing fibers have been found in the medulla, spinal cord, frontal and temporal cortex, and adjacent to the ependyma of the lateral ventricle (Nilaver et al., 1979). However, evidence that these neuronal tracts relate to CSF OT secretion remains to be developed.

The physiological significance of the OT rhythm in CSF remains unclear. CSF levels of the other posterior pituitary nonapeptide, arginine vasopressin (AVP), show a daily rhythm in the primate, cat, and sheep (Perlow et al., 1981; Reppert et al., 1981; H. G. Artman and D. A. Fisher, unpublished observations). In the monkey, the CSF AVP rhythm is synchronous with the CSF OT rhythm. In sheep, when both rhythms are manifested, they are nonsynchronous. Cats do not have an OT rhythm at all (S. M. Reppert, H. G. Artman, and D. A. Fisher, unpublished observations). It has been reported 


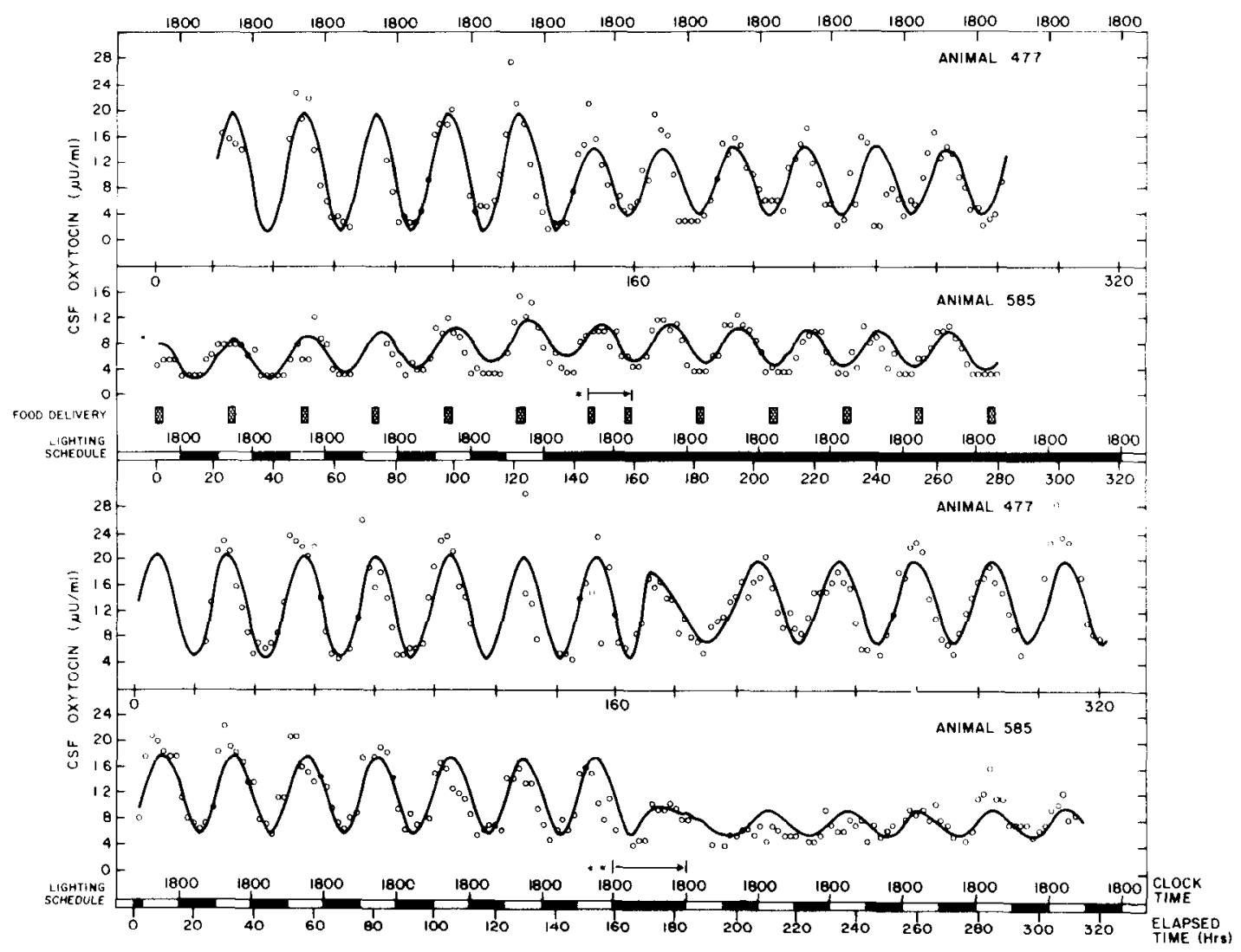

- 12 hour phase shift in food delivear

Figure 3. Variation of OT concentrations with time in two monkeys. Upper pair of curves, lighting and food schedule changes after $140 \mathrm{hr}$; lower pair of curves, lighting schedule shifted $12 \mathrm{hr}$ after 170 hr. The open circles are measured CSF OT concentrations. The solid lines show the computer-fitted sine curves generated for each animal and each treatment (see Tables I and II).

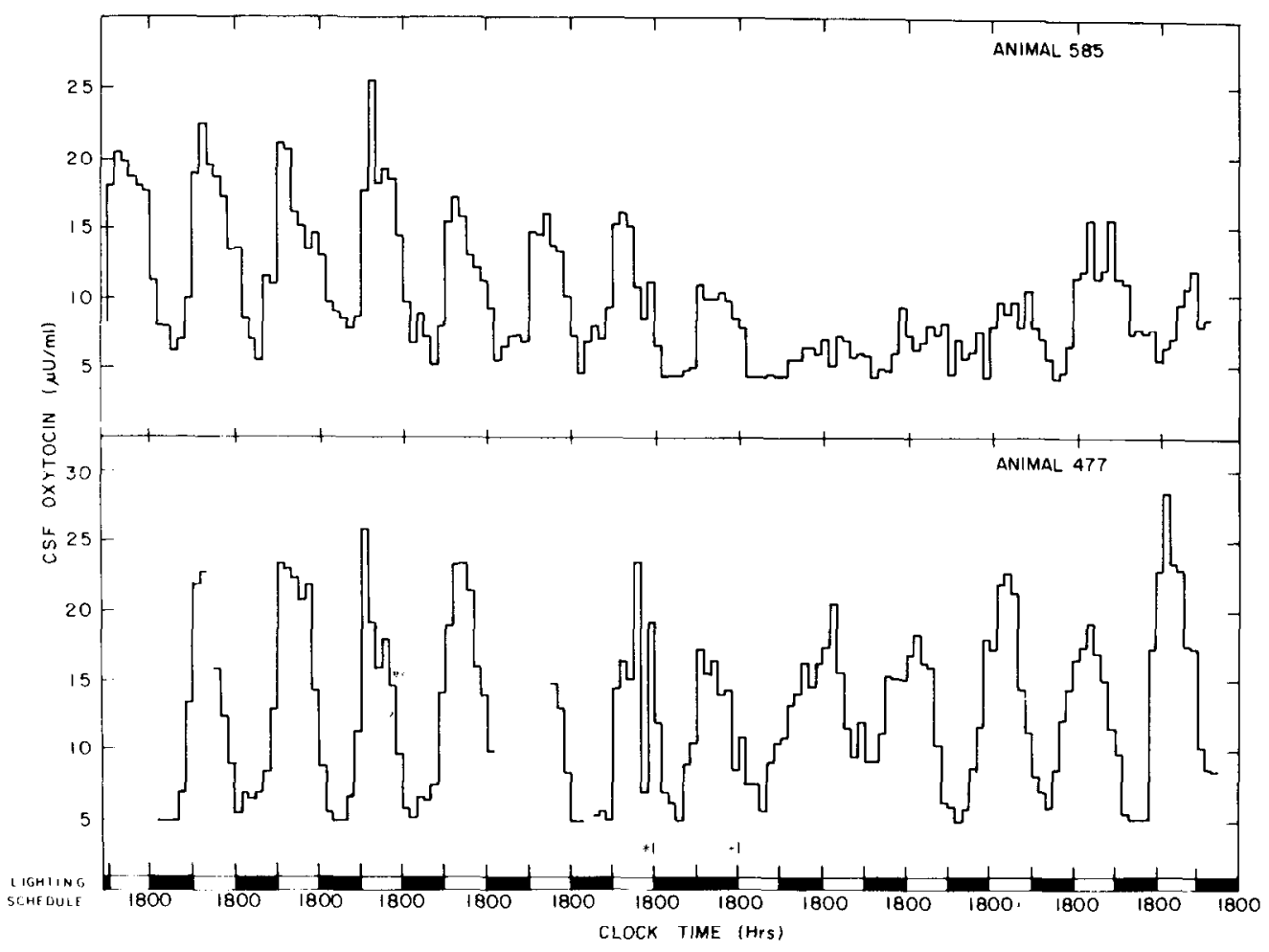

Figure 4. Pattern of CSF OT concentrations during a 12-hr phase shift of the diurnal lighting schedule. A phase reversal of the lighting schedule was accomplished by extending the normal dark period for an additional $12 \mathrm{hr}$. The periodic delivery of food and daily care also was phaseshifted from between 10 A.M. and 12 noon to 10 P.M. and 12 midnight coincident with the phase reversal in lighting. CSF was collected in 2-hr aliquots. 
that OT and AVP injected into the cerebral ventricles have opposite effects on memory (Bohus et al., 1978; Kovacs et al., 1979). This observation is puzzling in view of the synchrony of AVP and OT rhythms in the monkey. However, the memory studies were done in rats, and since the CSF patterns of the various neurohypophyseal rhythms seem to be species specific, the hormone functions in CSF also may vary with species. Clearly, further studies of the sources, control, and roles of the neurohypophyseal peptides in the CSF are warranted.

\section{Appendix}

Equations used for computer curve fitting. Let $x=$ time (hours) from the beginning of sample collection, $y=\mathrm{OT}$ concentration (microunits per $\mathrm{ml}$ ) in CSF sample, $A=$ mean level of $y$ over a full diurnal cycle, $B=$ amplitude of the variation of $y$ above and below $A, O=$ phase angle (radians) of the oscillations of $y$, and $T=$ period (hours) of the oscillations.

A simple sine function with fixed parameters is represented by

$$
y=A+B \sin [(2 \pi x / T)+O]
$$

Functions in which $A$ or $B$ changes linearly with $x$, with a coefficient $E$, are represented by either

$$
y=A+E x+B \sin [(2 \pi x / T)+O]
$$

or

$$
y=A+(B+E x) \sin [(2 \pi x / T)+O]
$$

while a linear change of the period $T$ gives

$$
y=A+B \sin [\{2 \pi x /(T+E x)\}+O]
$$

An alternative to equation 4 is to let $O$ change exponentially from an initial value $O_{1}$ to $O_{2}$, using

$$
y=A+B \sin \left[(2 \pi x / T)+O_{2}+\left(O_{1}+O_{2}\right) E^{G\left(x-x_{0}\right)}\right]
$$

where $x_{0}$ is the initial time when the change in $O$ begins and $G$ is a constant. For the OT data in this study, equation 2 or 3 fitted the results slightly better than equation 1 , but for any one of these three equations, the position of the peak values of $y$ is determined substan- tially by $T$ and $O$. However, for cases where the spacing of the peaks was changing, equation 5 gave substantially better results than equation 4 as might be expected intuitively. The equations used, estimated parameter values, and peak $y$ positions for the data in this study are shown in Table I.

\section{References}

Bohus, B., G. L. Kovacs, and D. DeWied (1978) Oxytocin, vasopressin and memory: Opposite effects on consolidation and retrieval processes. Brain Res. 157: 414-417.

Kovaes, G. L., B. Bohus, D. H. G. Versteeg, E. R. DeKloet, and D. DeWied (1979) Effect of oxytocin and vasopressin on memory consolidation: Site of action and catecholaminergic correlates after local microinjection with limbic-midbrain structures. Brain Res. 175: 303-314.

Nilaver, G., D. L. Hoffman, and E. A. Zimmerman (1979) Multiple projections of the magnocellular hypothalamus to other brain regions including cerebral cortex. Trans. Am. Neurol. Assoc. 104: 205-208.

Perlow, M. J., S. M. Reppert, H. G. Artman, and S. M. Seif (1981) Oxytocin, vasopressin, vasotocin and estrogen-stimulated neurophysin: Daily pattern in plasma and cerebrospinal fluid concentrations and response to estrogen stimulation. In Proceedings of the 63rd Annual Meeting of The Endocrine Society, Cincinnati, Ohio, June 1981, p. 272, The Endocrine Society, Bethesda, MD.

Reppert, S. M., M. J. Perlow, L. Tamarkin, and D. C. Klein (1979) A diurnal melatonin rhythm in primal cerebrospinal fluid. Endocrinology 104: 295-301.

Reppert, S. M., H. G. Artman, S. Swaminathan, and D. A. Fisher (1981) Vasopressin exhibits a rhythmic daily pattern of secretion into cerebrospinal fluid but not into blood. Science 213: 1256-1257.

Scott, D. E., G. P. Kozlowski, and M. N. Sheridan (1974) Scanning electromicroscopy in the ultrastructural analysis of the mammalian cerebral ventricular system. Int. Rev. Cytol. 37: $349-388$.

Weitzman, R. E., T. H. Glatz, and D. A. Fisher (1978) The effect of hemorrhage and hypertonic saline upon plasma oxytocin and arginine vasopressin in conscious dogs. Endocrinology 103: 2154-2160.

Zucker, I. (1980) Light, behavior and biologic rhythms. In Neuroendocrinology, D. T. Krieger and J. C. Hughes, eds., pp. 93-101, Sinauer Associates, Inc., Sunderland, MA. 